\title{
Beitrag zum Problem der Energiemessung in aquatischen Ökosystemen: Mikrokalorimetrische Untersuchungen von Planktonalgen
}

\author{
Von H. R. Bürgi, E. Szabó und H. Bachmann \\ EAWAG, Dübendorf \\ Manuskript eingegangen am 8. Mai 1981
}

\begin{abstract}
Contribution to the problem of energy measurement in aquatic ecosystems: Microcalorimetric studies of planktonic algae

The energy content from a batch culture of Pediastrum boryanum was measured in a Differential Scanning Calorimeter as combustion enthalpy. A clear linear relation exists between dry weight and energy content. The energy content in the exponential growth phase is constant, approx. $22.1 \mathrm{~kJ} / \mathrm{g} \mathrm{dry}$ weight (not ashfree).

The proportion of various organic substances - documented by different peaks in the thermograms change in time, apparently due to increasing growth-limitation by $\mathrm{P}$ and $\mathrm{N}$.
\end{abstract}

\section{Einleitung und Zielsetzung}

Die Analyse funktioneller Zusammenhänge in aquatischen Ökosystemen verlangt neben der Kenntnis über Stoffkreisläufe auch quantitative Daten des gerichteten Energieflusses. Dieser führt in Form chemischer Energie von einer Trophiestufe zur nächsten.

Die Art und Menge der chemischen Energieträger wird durch die ökologischen Faktoren beeinflusst und variiert entsprechend den physiologischen Eigenschaften von Art zu Art.

Der Energiegehalt der Organismen hängt von dieser chemischen Zusammensetzung ab: von ca. $17200 \mathrm{~J} \mathrm{~g}^{-1}$ für Kohlenhydrate bis ca. $39800 \mathrm{~J} \mathrm{~g}^{-1}$ für die energiereichsten Verbindungen wie Fette [1]. Damit lässt sich erklären, dass nur sehr aufwendige chemische Analysen der verschiedenen Stoffgruppen oder die direkte kalorimetrische Messung der Verbrennungsenthalpie zu befriedigenden Resultaten führen kann.

Die bisher verwendeten direkten Messverfahren mittels Bombenkalorimeter oder Nassoxidation sind umständlich und teilweise schlecht interpretierbar. Bei dem Bombenkalorimeter tauchten noch zusätzliche Schwierigkeiten auf, da neben dem Wärmeaustausch auch Druck- bzw. Volumenänderungen (Kompressionsenergie) zu messen sind. 
Mit der Entwicklung schnell arbeitender und empfindlicher DSC-Geräte (Differential Scanning Calorimeter) ist es möglich geworden, mehrere Proben an einem Arbeitstag routinemässig zu verarbeiten. Die Empfindlichkeit der Messung liegt je nach Gerätetyp im Grössenbereich von 1 bis $30 \mu \mathrm{W}$ und erlaubt z. B. die Messung des Energieinhaltes einzelner Daphnien. Gnaiger [3] verwendet solche Geräte zur Bestimmung der Wärmeproduktion bei der Respiration durch aquatische Kleintiere.

Über die Anwendung der Kalorimetrie in der Biologie geben Lamprecht und Schaarschmidt [6] eine Zusammenstellung. Cummins und Wuycheck [1] haben in einer Literaturübersicht die Energieäquivalente vieler Organismen (auf verschiedener Basis gemessen) tabelliert. Auf die vielseitigen Anwendungen der Mikrokalorimeter in der Chemie (Reinheitsbestimmungen, Kunststoffpolymerisation, Sicherheitsanalysen für exotherme Prozesse usw.), soll hier nicht weiter eingegangen werden, zumal darüber ein grosses Schrifttum existiert (vgl. Thermochimica Acta). Zielsetzung dieser Arbeit war:

1. Erarbeitung einer Methodik zur Energieinhaltsbestimmung geringster Mengen von Planktonorganismen.

2. Abklärung des Einflusses variierter Nährstoffbedingungen auf die Relation von Energie pro Biomasse.

3. Studium der energetischen Wechselwirkung zwischen Organismus und organischen Inhaltsstoffen der Kulturlösung.

\section{Material und Methoden}

\subsection{Versuchsanordnung und Kulturtechnik}

In zwei Vorversuchen mit Batchkulturen wurden Tiegel- und Filtermaterialien zur Bestimmung der Verbrennungsenthalpie geprüft; dem dritten Versuch lag die oben erwähnte Zielsetzung zugrunde.

Kulturorganismus: Die Versuche wurden mit einer nicht axenischen Reinkultur von Pediastrum boryanum betrieben. Dieser Testorganismus wurde gewählt, weil er im Sommerplankton eutropher Seen zuweilen häufig vertreten ist und unter nicht nährstofflimitierten Bedingungen keine Gallerte ausscheidet, wodurch die Filtration grösserer Biomassen durch Silbermembranfilter möglich ist. $P$. boryanum wurde von Gavrieli [2] aus dem Greifensee isoliert und in Chemostatkulturen physiologisch charakterisiert.

Kulturbedingungen: Batchkulturen mit 101 Duran ${ }^{\circledR}$-Enghals-Flaschen wurden in thermisch stabilen Kellerräumen bei $24^{\circ} \mathrm{C}$ im Tag-Nacht-Rhythmus (16:8 Std.) gehalten. Lichtquelle: Philips-«white light»-Leuchtstoffröhren in einer Anordnung, die in der vertikalen Achse des Kulturgefässes eine Leistung von ca. $20 \mathrm{Watt} / \mathrm{m}^{2}$ lieferten. Rühren mit Teflon-Magnetrührer. Die Kultur wurde mit Gasgemisch (Luft mit ca. $3 \% \mathrm{CO}_{2}$ ) durchblasen $(50 \mathrm{ml} / \mathrm{min}$ ). Damit konnte die Kultur kontinuierlich mit Kohlenstoff versorgt werden. Weiter wurde dadurch die unerwünschte Erhöhung des $\mathrm{pH}$-Wertes verhindert, welche sonst als Folge der intensiven Assimilation bzw. $\mathrm{CO}_{2}$ - und Bikarbonataufnahme eintreten müsste. 
Kulturmedium: Künstliches Greifenseewasser (Ionenbilanz gemäss Greifensee) nach Fricker (in Vorb.) mit 1,29 mg Orthophosphat, 9,15 mg Nitrat und 58,3 mg anorganischem Kohlenstoff pro Liter.

Probenahmen: Jeweils 15 Minuten vor Ablauf der Hell- bzw. Dunkelphase wurden ca. $100 \mathrm{ml}$ Suspension aus der Kultur in ein verdunkeltes Gefäss abgefüllt.

Parameter: Die Proben wurden auf folgende Komponenten untersucht:

- Aus der Suspension unmittelbar bestimmt:

Trockengewicht $(6 \times 10 \mathrm{ml})$. Diese Proben wurden nach dem Trocknen und Wägen zur Bestimmung des Energieinhaltes (als negative Verbrennungsenthalpie gemessen) verwendet.

Zelldichte und daraus durch Umrechnung anhand der Zellgrösse BiomasseNassgewichte.

Gesamtphosphor (3-5 ml) und $\mathrm{pH}$-Wert.

- Aus dem Filtrat bestimmt:

Orthophosphat und UV-Phosphat [11] $(3 \times 5 \mathrm{ml})$.

Gesamtphosphor gelöst $(3 \times 5 \mathrm{ml})$.

Nitrat-Stickstoff $(3 \times 5 \mathrm{ml}$, gegen Versuchsende $3 \times 10 \mathrm{ml})$.

$T I C$, darauf folgend DOC $(3 \times 5 \mathrm{ml})$. Die in Klammer angegebenen Zahlen bedeuten die Anzahl der Wiederholungen und das Volumen der verwendeten Proben.

\subsection{Methoden}

Trockengewicht: Die sechs Parallelproben wurden auf «Flotronics»-Silbermembranfilter $(1,2 \mu \mathrm{m}, \varnothing 13 \mathrm{~mm})$ abfiltriert. Danach wurden die Filter bei $40^{\circ} \mathrm{C}$ im Vakuum getrocknet und über Silicagel im Exsikkator aufbewahrt. Die Silbermembranfilter wurden vor der Verwendung in einem Muffelofen bei $400^{\circ} \mathrm{C} 2$ Stunden lang erhitzt, um Fremdverunreinigungen zu eliminieren.

Biomasse: Zählung in Röhrenverbundkammern nach Utermoeh1 [14] und Multiplikation mit Volumenfaktoren, gemittelt für verschiedene Grössenklassen von Kolonien.

Phosphorbestimmung: Die kleinen Probemengen erforderten eine spezielle chemische Analysentechnik. Hier können vorteilhaft automatische Methoden eingesetzt werden, welche zudem äusserst genaue Daten liefern. Sämtliche Phosphorverbindungen wurden mit einem Technicon-Autoanalyzer bestimmt [11]:

- Orthophosphat-Ion (oP) als Heteropolymolybdänblau nach Reduktion mit Ascorbinsäure [15].

- UV-Phosphat (UV-P) mittels Kaliumperoxodisulfat und Photooxidation, danach als Orthophosphat-Ion bestimmt [11].

- Gesamtphosphor: Aufschluss mittels Kaliumperoxodisulfat im Autoklaven [11], Bestimmung als Orthophosphat-Ion.

Aus jeder Probe wurden also vier Phosphoranalysen durchgeführt.

Die Resultate erlauben folgende Gruppentrennung:

oP: Orthophosphat-Ion, wurde als $\mathrm{KH}_{2} \mathrm{PO}_{4}$ vorgelegt und dient als Phosphorquelle der Algen. 
COP: Die organischen, gelösten Phosphorverbindungen werden durch UV-Oxidation (Photooxidation) in oP überführt (Differenz zwischen UV-P und oP).

POP: Kondensierte, gelöste Phosphorverbindungen werden durch UV-Oxidation nicht, durch Hydrolyse (Säure und erhöhte Temperatur) in oP umgewandelt (Differenz zwischen Gesamt-P gelöst und UV-P). Die COP- und POP-Phosphate [16] sind Autolyse- und Ausscheidungsprodukte der Algen.

PP: Partikulärer Phosphor, inkorporiert in die Zellen (Differenz zwischen Gesamt-P und Gesamt-P gelöst).

Nitrat-Stickstoff wurde nach der Salicylat-Methode kolorimetrisch bestimmt [8].

Gesamter anorganischer Kohlenstoff $(\mathrm{TIC}=$ total inorganic carbon) und gelöster organischer Kohlenstoff ( $\mathrm{DOC}=$ dissolved organic carbon) wurden mit einer kontinuierlichen, automatisierten Methode bestimmt [12]. Durch Säurezugabe werden die Karbonate und Hydrogenkarbonate in Kohlensäure überführt, mit Stickstoffgas aus der Lösung entfernt und in einem IR-Messgerät analytisch erfasst (TIC). Die Probe enthält nun keinen anorganischen Kohlenstoff mehr. Sie wird mit Kaliumperoxodisulfat versetzt, photooxidiert und die jetzt entstandene Kohlensäure wieder mit Stickstoffgas aus der Lösung ausgetrieben. In einem zweiten IR-Messgerät wird dieser Anteil (= DOC) bestimmt.

Energiemessung (Mikrokalorimetrie): Die Messung erfolgte mit einem DSC-Gerät der Firma Setaram. Bei dieser Methode ist es wichtig, dass im Probenkanal und Referenzkanal ungefähr gleich schwere Proben mit gleichem $C_{p}$ (spezifische Wärme) gemessen werden. Dies setzt eine genaue Tarierung der leeren Tiegel und Filter voraus. Vorversuche wurden mit verschiedenen Tiegeltypen und Filtermaterialien durchgeführt. Die besten Resultate lieferten Platintiegel mit perforiertem Deckel und «Flotronics»-Silbermembranfilter, welche sehr gewichtskonstant sind und keine Materialverluste beim Manipulieren ergaben.

Die sechs Replica wurden in je zwei gewichtsgleiche Parallelproben gruppiert. Zur Gewinnung der Basislinie wurde je eine Probe unter Argon-Atmosphäre programmiert $\left(10^{\circ} / \mathrm{min}\right)$ aufgeheizt (Zimmertemperatur bis $550^{\circ} \mathrm{C}$, mit einer zwischengeschalteten isothermen Phase bei $100^{\circ} \mathrm{C}$ ) und die Messdaten im Computer gespeichert. Die anderen Parallelproben wurden sodann mit dem gleichen Temperaturprogramm unter $\mathrm{O}_{2}$-Atmosphäre (Spülung $25 \mathrm{ml} / \mathrm{h}$ ) verbrannt. Die Verbrennungskurve wurde mit der gespeicherten Basislinie korrigiert und die Verbrennungsenthalpie berechnet. In der Referenzkammer wurde stets der gleiche Platintiegel mit leerem Filter positioniert. Die Eichung erfolgte durch Verbrennung von Glucose.

Durch Verrechnung der Argon-Basislinie mit der Verbrennungskurve ist es möglich, allfällige endotherme Peaks (z.B. von Verdampfung), welche einen Teil der Verbrennungswärme aufbrauchen und damit maskieren, zu subtrahieren.

Da bei der Verbrennung gasförmige Produkte entstehen, welche den Tiegel verlassen, sinkt im Laufe der Aufheizphase die Probenmenge, was einen Grundliniensprung bewirkt. Bei der Integration der Enthalpie muss dieser Vorgang berücksichtigt werden. In den vorliegenden Versuchen wurden Anfangs- und Endpunkt der Verbrennungskurve durch eine Gerade verbunden. Der Fehler, welcher bei der Berechnung der Fläche dadurch entstand, wurde durch planimetrische Messung ermittelt; er liegt stets unter $1 \%$. 


\section{Resultate und Diskussion}

\subsection{Zeitreihen der Nährstoffe in der Kulturlösung}

Die chemischen Analysenresultate sind in Tabelle 1 zusammengefasst. Für die Algen wird ein atomares Gewichtsverhältnis der Hauptnährstoffe $(\mathrm{P}, \mathrm{N}, \mathrm{C})$ von 1:16:106 als ideal betrachtet (Redfield et al. [9]). Durch Aufbruch der P- und NReserven in der Pediastrum-Kultur verschob sich dieses Verhältnis in der Lösung von 1:16:77 am Anfang zu 1:18:9671 nach einer Woche. Phosphor und Stickstoff nahmen in der Kulturlösung in einer sigmoiden Kurve synchron ab. Nach 168 Stunden betrug die Phosphat(oP)-Konzentration nur noch 0,3\% des Ausgangswertes. Damit war praktisch eine Wachstumslimitierung der Kultur durch $\mathrm{P}$ und $\mathrm{N}$ gegeben. Mit zunehmender Zelldichte ist ein Ansteigen gelöster organischer P- und C-Verbindungen zu beobachten. Der DOC (gelöster organischer Kohlenstoff) weist dabei deutlich Tag-Nacht-Schwankungen auf. Mit einer Ausnahme liegt der Wert nach der Hellphase deutlich höher als der Dunkelwert.

Die Stabilisierung des pH war nur in Grenzen möglich, da der Fluss von $\mathrm{CO}_{2}$-Gas erst nach Vorliegen der TIC-Messungen adaptiert werden konnte und keine unmittelbare Erfolgskontrolle zuliess.

Wird anhand der PP-Anteile nach der Redfield-Formel auf die Biomasse umgerechnet, ergibt sich innerhalb der ersten 144 Stunden ein exponentieller Verlauf des errechneten Trockengewichts, gefolgt von einem Plateau der maximal nach dieser

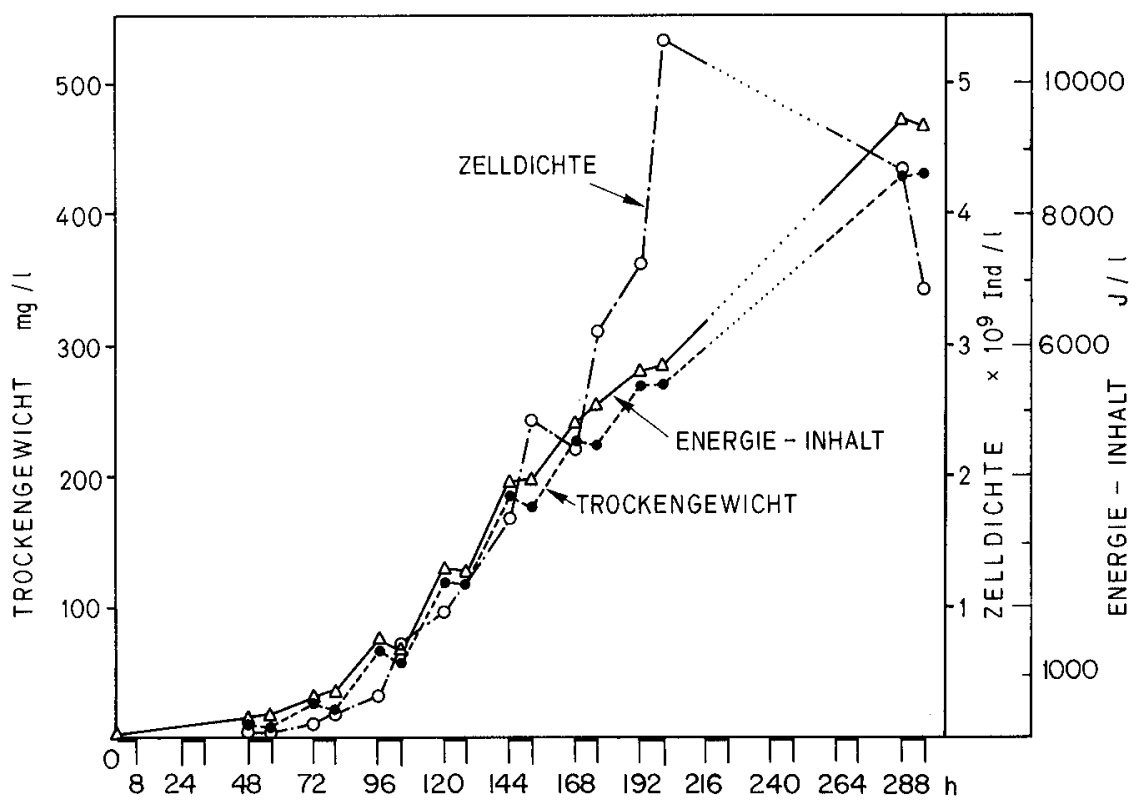

Abb. 1. Die zeitliche Entwicklung der Zelldichte, Biomasse (Trockengewicht) und des Energieinhaltes einer Pediastrum-Kultur im Batchverfahren.

Figure 1. The temporal development of cell density, biomass (dry weight) and caloric content of a Pediastrum batch culture. 
Tabelle 1. Chemische Analysen der Nährlösung.

Table 1. Chemical analysis of the culture medium.

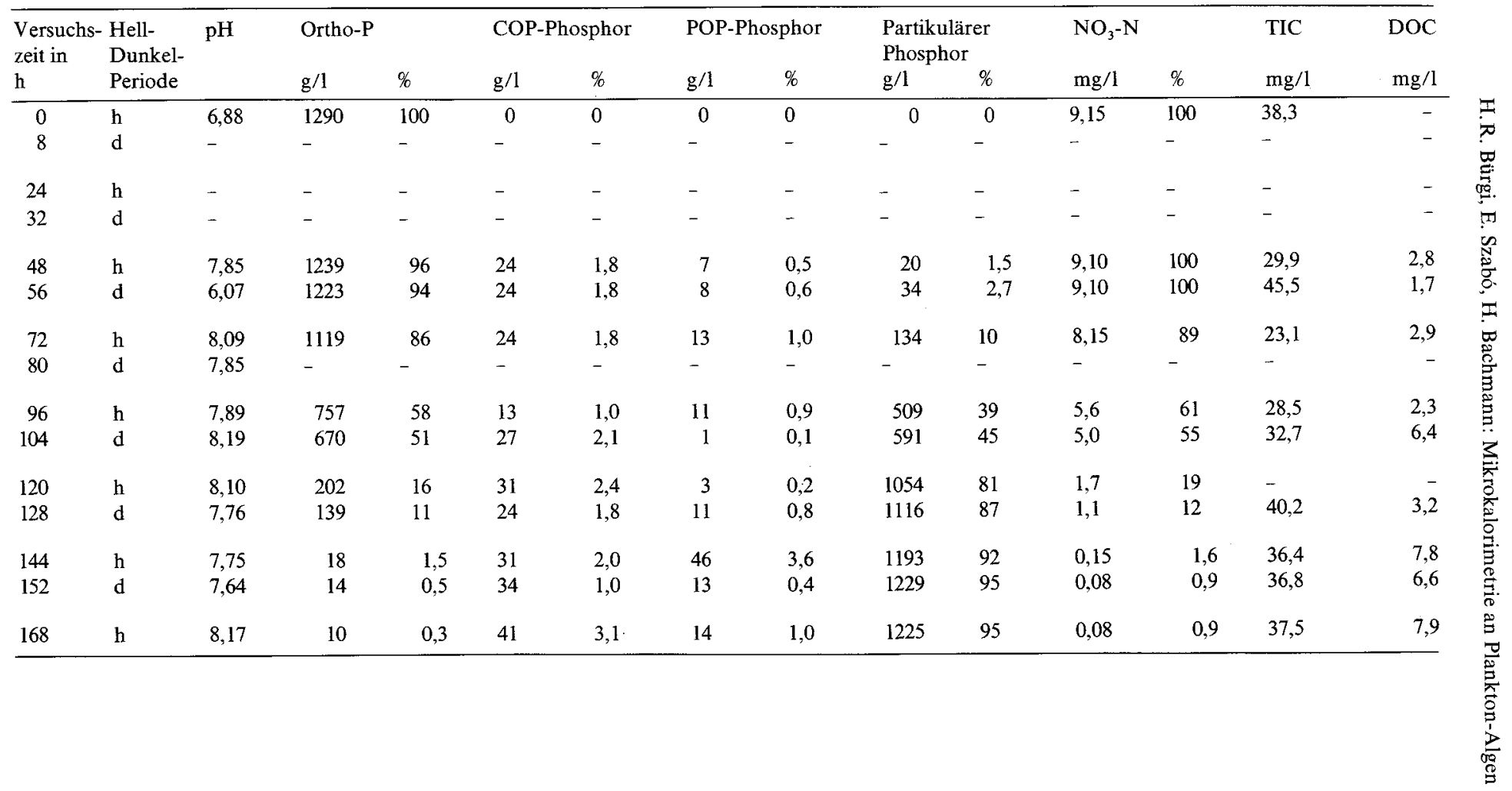


Formel erreichbaren Biomasse von $101 \mathrm{mg}$ pro Liter (entsprechend den $1290 \mu \mathrm{g}$ Gesamtphosphor in der Lösung). Demgegenüber nimmt die tatsächlich gemessene Trockensubstanz bis zum Abbruch des Experimentes nach 296 Stunden noch kontinuierlich zu. Die mit dem vorhandenen P-Pool realisierbare Biomasse liegt demnach 4-5mal höher als die nach der Redfield-Formel errechnete. Gavrieli [2] fand in Chemostatversuchen für Pediastrum-Kulturen unter P-Limitierung einen PGehalt des Trockengewichts von 1 bis $3 \%$, was einem atomaren $\mathrm{P}: \mathrm{C}$-Verhältnis von 1:900 bis 1:300 entspricht.

\subsection{Zeitreihen der Biomasse und des Energieinhaltes (Tab. 2 und Abb. 1)}

In der ersten Phase des Versuchs (bis 144 Std.) herrschten vom Nährstoffangebot her günstige Bedingungen; die Trockensubstanz nahm exponentiell zu; die Wachstumsrate betrug optimal $0,0427 \mathrm{~h}^{-1}$. Mit dem Einsetzen der Nährstofflimitierung und einer wirksamen Selbstbeschattung folgte zunächst noch ein lineares Wachstum und schliesslich der Zusammenbruch der Kultur, was anhand des Farbumschlages von Grün zu Ocker zu erkennen war und der von einer rasanten Zunahme des Bakterienwachstums begleitet wurde.

Die Zelldichte nahm auch nach dem Aufbrauch der P- und N-Nährstoffe noch zu, wobei aber aus der Teilung sukzessive kleinere Kolonien mit kleineren Zellen hervorgingen. Trockengewicht und Energieinhalt nahmen auch nach dem Zusammenbruch der Zelldichte noch schwach zu, was aber auch auf Anhäufung toter organischer Substanz (insbesondere leere Zellhüllen) zurückzuführen ist. Die Zelldichte nahm in der Dunkelphase stärker zu als im Licht. Der Trockengewichtsanteil der analysierten Biomasse (Nassgewicht) lag im Mittel bei 21\% (vgl. Tab.2).

Die Entwicklung der Trockensubstanz verlief umgekehrt: die Zunahme erfolgte nur während der photosynthetisch wirksamen Hellphase. Die leichte Abnahme des Trockengewichts während der Nacht wurde nicht von einem entsprechenden Verlust an Energie begleitet, sondern diese blieb während der Dunkelphase mehr oder weniger konstant.

Tabelle 2a. Analysen der Biomasse- und Energieparameter der Hellwerte.

Table $2 \mathrm{a}$. Analysis of the biomass, dry weight, cell density and caloric content at the end of light phase.

\begin{tabular}{|c|c|c|c|c|c|c|c|c|c|c|}
\hline \multirow{3}{*}{$\begin{array}{l}\text { Versuchs- } \\
\text { zeit in } \\
\mathbf{h}\end{array}$} & \multicolumn{3}{|c|}{ Trockengewicht/1 } & \multicolumn{2}{|c|}{ Biomasse } & \multirow{3}{*}{$\begin{array}{l}\begin{array}{l}\text { Zell- } \\
\text { dichte }\end{array} \\
10^{9} / 1\end{array}$} & \multicolumn{3}{|c|}{ Energie/l } & \multirow{3}{*}{$\begin{array}{r}\text { Energie } \\
\mathrm{kJ} / \mathrm{g}\end{array}$} \\
\hline & \multirow{2}{*}{$\begin{array}{l}\overline{\mathrm{x}} \\
\mathrm{mg} \\
\end{array}$} & \multicolumn{2}{|c|}{ Abweichung } & \multirow{2}{*}{$\begin{array}{l}\text { Nass- } \\
\text { gewicht } \\
\text { mg }\end{array}$} & \multirow{2}{*}{$\begin{array}{l}\text { berech- } \\
\text { net }^{*} \text { ) } \\
\mathrm{mg}\end{array}$} & & & $\mathrm{Abv}$ & lung & \\
\hline & & $\pm \mathrm{mg}$ & $\%$ & & & & $\mathrm{~J}$ & $\pm \mathbf{J}$ & $\%$ & \\
\hline 48 & 9,5 & 1,6 & 16,8 & 41 & 1,7 & 0,055 & 285 & 25 & 8,8 & 30 \\
\hline 72 & 26,8 & 0,7 & 2,6 & 82 & 10,5 & 0,099 & 635 & 43 & 6,7 & 23,6 \\
\hline 96 & 64,1 & 0,8 & 1,3 & 207 & 39,9 & 0,32 & 1562 & 71 & 4,5 & 24,4 \\
\hline 120 & 118,4 & 1.8 & 1,5 & 549 & 82,5 & 0,95 & 2639 & 20 & 0,7 & 22,3 \\
\hline 144 & 182,3 & 2,3 & 1,3 & 1050 & 93,4 & 1,67 & 4015 & 133 & 3,3 & 22,0 \\
\hline 168 & 225,4 & 6,2 & 2,8 & 1130 & 95,4 & 2,22 & 4903 & 28 & 2,2 & 21,8 \\
\hline 192 & 270,0 & 8,3 & 3,1 & 1820 & $\max$ & 3,60 & 5785 & 172 & 2,4 & 21,5 \\
\hline 288 & 429,8 & 2,2 & 0,5 & 2444 & $\begin{array}{l}\text { möglich } \\
101\end{array}$ & 4,3 & 9731 & 310 & 3,2 & 22,6 \\
\hline
\end{tabular}

*) Biomasse berechnet nach Redfield [9]: $1 \mathrm{~mol} P=2426 \mathrm{~g}$ Biomasse; $1 \mu \mathrm{g} \mathrm{P}=78,32 \mu \mathrm{g}$ Biomasse (Trockengewicht) (nach dieser Formel ist die maximal erreichbare Biomassse $101 \mathrm{mg} / 1290 \mu \mathrm{g}$ P). 
Tabelle 2b. Analysen der Biomasse- und Energieparameter der Dunkelwerte.

Table $2 \mathrm{~b}$. Analysis of the biomass, dry weight, cell density and caloric content at the end of dark phase.

\begin{tabular}{|c|c|c|c|c|c|c|c|c|c|c|}
\hline \multirow{3}{*}{$\begin{array}{l}\text { Versuchs- } \\
\text { zeit in } \\
\mathrm{h}\end{array}$} & \multicolumn{3}{|c|}{ Trockengewicht/1 } & \multicolumn{2}{|l|}{ Biomasse } & \multirow{3}{*}{$\begin{array}{l}\text { Zell- } \\
\text { dichte } \\
10^{9} / 1\end{array}$} & \multicolumn{3}{|c|}{ Energie/1 } & \multirow{3}{*}{$\begin{array}{r}\text { Energie } \\
\mathrm{kJ} / \mathrm{g} \\
\end{array}$} \\
\hline & \multirow{2}{*}{$\begin{array}{l}\bar{x} \\
m g\end{array}$} & \multicolumn{2}{|c|}{ Abweichung } & \multirow{2}{*}{$\begin{array}{l}\text { Nass- } \\
\text { gewicht } \\
\text { mg }\end{array}$} & \multirow{2}{*}{$\begin{array}{l}\text { berech- } \\
\text { net*) } \\
\text { mg }\end{array}$} & & & Abw & 10 & \\
\hline & & $\pm \mathrm{mg}$ & $\%$ & & & & $\mathrm{~J}$ & $\pm \mathrm{J}$ & $\%$ & \\
\hline 56 & 7,7 & 0,5 & 6,5 & 39 & 2,6 & 0,062 & 329 & 16 & 4,9 & 42,8 \\
\hline 80 & 24,9 & 2,0 & 8,0 & 104 & - & 0,225 & 693 & 30 & 4,3 & 27,9 \\
\hline 104 & 56,2 & 2,4 & 4,3 & 290 & 46,3 & 0,720 & 1384 & 87 & 6,3 & 24,6 \\
\hline 128 & 118,9 & 1,6 & 1,4 & - & - & & 2607 & 93 & 3,6 & 22,0 \\
\hline 152 & 175,8 & 1,2 & 0,7 & 1010 & 96,2 & 2,440 & 4059 & 144 & 3,6 & 23,1 \\
\hline 176 & 223,2 & 1,4 & 0,6 & 1270 & - & 3,100 & 5235 & 207 & 4,0 & 23,5 \\
\hline 200 & 268,2 & 1,4 & 0,5 & 2200 & - & 5,360 & 5838 & 41 & 0,7 & 21,8 \\
\hline 296 & 432,6 & 3,4 & 0,8 & 1650 & - & 3,400 & 9605 & 161 & 1,7 & 22,1 \\
\hline
\end{tabular}

*) Biomasse berechnet nach Redfield [9]: $1 \mathrm{~mol} \mathrm{P}=2426 \mathrm{~g}$ Biomasse; $1 \mu \mathrm{g} P=78,32 \mu \mathrm{g}$ Biomasse (Trockengewicht) (nach dieser Formel ist die maximal erreichbare Biomassse $101 \mathrm{mg} / 1290 \mu \mathrm{g} \mathrm{P}$ ).

Die Veränderung des Verhältnisses von Energieinhalt zu Trockengewicht ist nur möglich, wenn sich die Energieträger umwandeln, wobei wahrscheinlich zum Teil die Energie für den Stoffwechsel in der Dunkelphase durch Aufnahme der gelösten organischen Verbindungen (DOC) aus der Nährlösung geschöpft werden kann. Die deutlichen Tag-Nacht-Schwankungen des DOC (Tab.1) dürften diese Interpretation bestätigen.

\subsection{Relation zwischen Trockengewicht und Energieinhalt}

Zwischen Trockengewicht $\mathrm{X}$ und Energieinhalt $\mathrm{Y}=$ negative Verbrennungsenthalpie) besteht eine gute Korrelation (Abb.2).

Regressionsgerade: $\mathrm{Y}=\mathrm{A}+\mathrm{B} \cdot \mathrm{X}$.

A: 0,92, B: 22,21, Bestimmtheitsmass: 0,997.

Dabei ist zu berücksichtigen, dass die Relation Energie zu Trockengewicht im TagNacht-Rhythmus schwankt (siehe oben!). Die Regressionsgerade geht nicht durch den Nullpunkt.

Bei niedrigen Zelldichten ist der Energiegehalt der Trockensubstanz leicht erhöht (Abb.3). Wieweit dieser Effekt auf methodischen Fehlern beruht, muss noch abgeklärt werden; es wäre aber auch denkbar, dass die frisch überimpften Zellen durch den Milieuwechsel der Kultur zunächst in der Teilung gehemmt sind (lagPhase) und dadurch weniger gespeicherte Energie veratmet wird.

Als Mass der Streuung wurde wegen der geringen Zahl von Parallelproben die mittlere absolute Abweichung berechnet (Tab.2a und b). Diese Streuung liegt für die Energiedaten im gleichen Bereich wie bei den Trockengewichtsbestimmungen mittels Mikrowaage. Im unteren Bereich ist die Energiemessung sogar noch etwas besser, während die prozentuale Streuung bei grösseren Substanzmengen beim Wägeprozess geringer wird.

Die tatsächlich eingewogenen Mengen belaufen sich auf 0,07 bis 2,2 $\mathrm{mg}$, mit einer Energie von 3,4 bis $48 \mathrm{~J}(0,81-11,5 \mathrm{cal})$. Unter Berücksichtigung der absoluten Streuung ergibt sich eine Empfindlichkeitsgrenze von ca.0,167 J (0,04 cal). Da die 


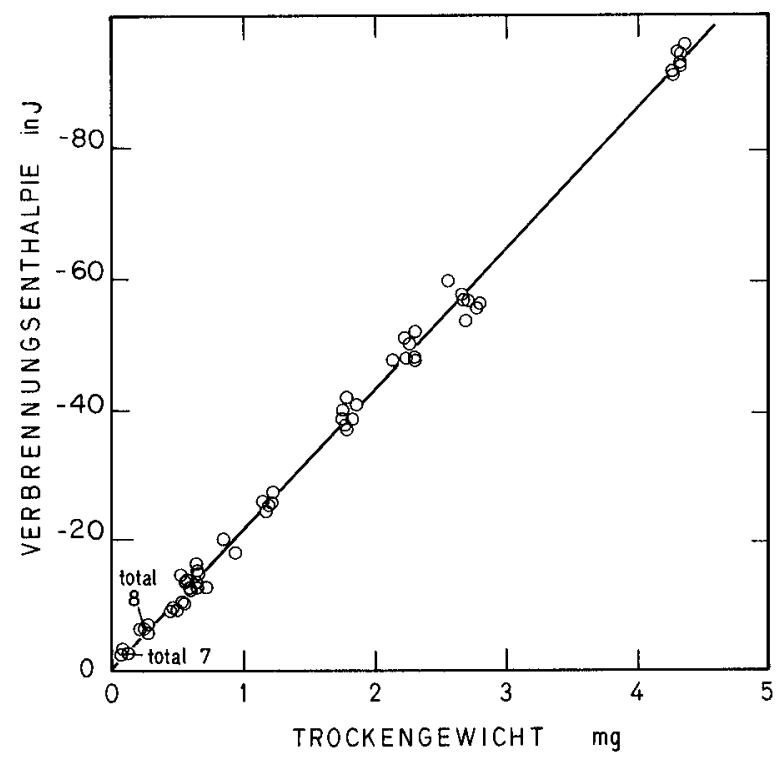

Abb.2. Beziehung zwischen Verbrennungsenthalpie und Biomasse (Trockengewicht).

Figure 2. Relation between combustion enthalpy and biomass (dry weight).

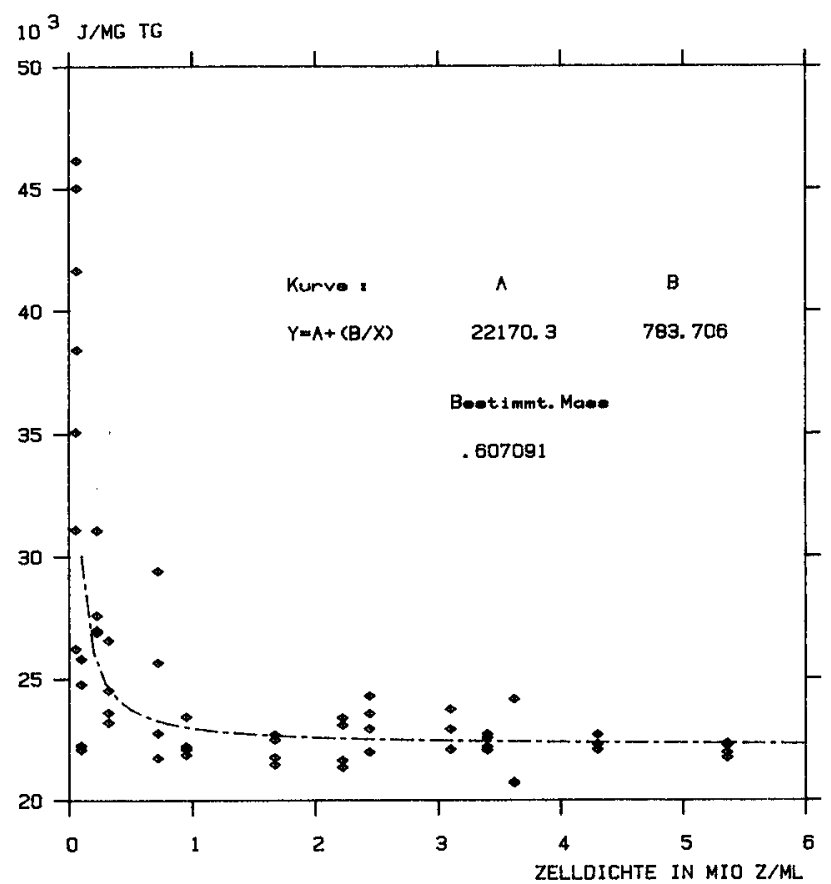

Abb.3. Beziehung zwischen dem spezifischen Energieinhalt ( $J$ pro $\mathrm{mg}$ Trockengewicht) und der Zelldichte der Algenkultur.

Figure 3. Relation between the specific energy content (J per mg dry weight) and the cell density. 
Empfindlichkeit des Gerätes noch weit tiefer liegt, dürfte aber durch Standardisierung der Probenahme und Filtration und durch Auswahl exakt gewichtsgleicher Tiegel und Filter eine weitere Verbesserung dieses an sich schon sehr guten Resultats möglich sein.

\subsection{Das Aufheizdiagramm}

\subsection{Basislinie}

Die unter Argon-Atmosphäre durchgeführte Messung weist alle thermischen Prozesse auf, welche nicht durch Verbrennung mit Sauerstoff bedingt sind. Die Kurve verläuft weitgehend gerade, was darauf zurückzuführen ist, dass die Proben unter Vakuum getrocknet wurden und wenig Wasser (Kristallwasser) enthielten. Die Verdampfung der organischen Substanz beginnt bei ca. $540 \mathrm{~K}$ (schwacher endother-

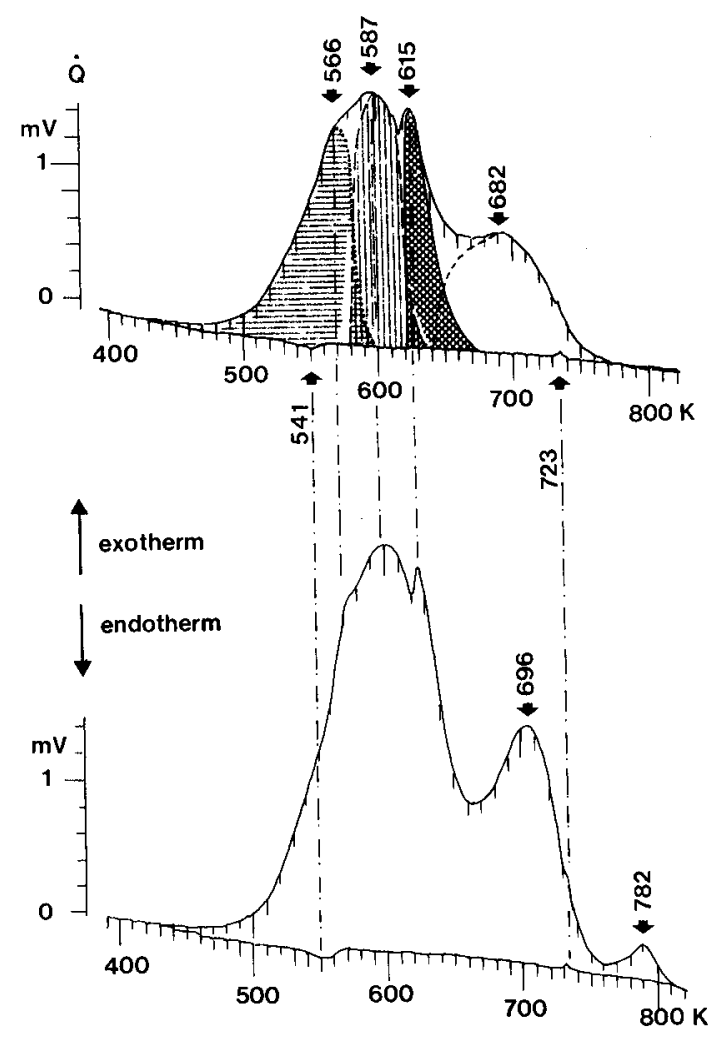

Abb. 4. Thermische Diagramme einer wachsenden (oben) und einer stagnierenden (unten) PediastrumKultur unter Argon-Atmosphäre (Basislinie) oder im $\mathrm{O}_{2}$-Fluss (Verbrennungsenthalpie).

Figure 4. Thermal diagrams of a growing (upper graph) and a stagnating (lower graph) Pediastrum culture in an argon atmosphere (baseline) or in oxygen stream (combustion enthalpy). 
mer Peak, siehe Abb.4). Bei $723 \mathrm{~K}^{1}$ ) ist bei allen verwendeten Silbermembranfiltern der Firma «Flotronic» ein schwacher exothermer Peak von 0,08 J (0,02 cal) zu beobachten. Die Untersuchung von Filtern im Raster-Elektronenmikroskop zeigte, dass dies die Folge einer Umwandlung der Filterstruktur zu grobporiger Form ist. Aus diesem Grund ist es wesentlich, dass die Filter bei der Vorbereitung nicht über $440^{\circ} \mathrm{C}$ erhitzt werden.

Der ziemlich gestreckte Verlauf der Basislinie weist auf keine Verdampfung anorganischer Salze (Karbonate usw.) hin, was im Temperaturtestbereich auch nicht zu erwarten war.

\subsection{Kurve der Verbrennungsenthalpie}

Der Kurvenverlauf der Messproben (Verbrennung mit $\mathrm{O}_{2}$ ) ergibt deutliche Peaks bei $587 \mathrm{~K}, 615 \mathrm{~K}$ und $682 \mathrm{~K}^{1}$ ) während der ganzen Versuchsdauer (Abb.4). Mehr oder weniger ausgeprägt erscheint eine Schulter bei 566 K. Bei der Probe nach 296 Stunden (beginnender Zusammenbruch infolge Nährstofflimitierung) erscheint konstant ein weiterer schwacher Peak bei $782 \mathrm{~K}$, während der Peak bei $682 \mathrm{~K}$ der früheren Proben enger und nach hinten verschoben ist ( $696 \mathrm{~K})$.

Die Verbrennung beginnt bei ca. $450 \mathrm{~K}$ und endet bei $800 \mathrm{~K}$. Die Energieanteile der einzelnen Peaks, deren Fläche in Abb. 4 graphisch herausgestellt wurden, betragen:

Peak 566: $29 \%$,

Peak 587: 27\%,

Peak 615: 26\%,

Peak 682: $18 \%$.

Die Breite der Peaks deutet auf Substanzgemische hin, die logischerweise bei Lebewesen vorhanden sind. Eine Zuordnung zu einzelnen Stoffgruppen ist schwierig. Am ehesten gelingt dies für den Peak bei $615 \mathrm{~K}$; dieser geht vermutlich auf die Verbrennung der Zellulose zurück [10].

\section{Zusammenfassung}

Es wurden eine mikrokalorimetrische Methode zur Messung der Verbrennungsenthalpie des Filterrückstandes einer Batchkultur der Alge Pediastrum boryanum getestet. Am Ende der Dunkel- bzw. Hellphase wurden jeweils sechs Parallelproben auf Silbermembranfilter filtriert. Zur Energiemessung wurden davon zwei unter Argon-Atmosphäre und vier im $\mathrm{O}_{2}$-Strom programmiert erhitzt.

Die Empfindlichkeit der Methode gestattet, die Energie von 0,07 mg Trockensubstanz auf $\pm 8 \%$ genau zu messen. Damit ist die Empfindlichkeit der Geräte noch nicht voll ausgeschöpft. Durch konsequente Standardisierung der einzelnen Manipulationen lässt sich eine noch bessere Auflösung erzielen.

Die Verbrennung der Substanzen beginnt bei $450 \mathrm{~K}$ und endet bei ca. $800 \mathrm{~K}$. Es sind

1) Die zeitliche Verzögerung der programmierten (Tp) von der reellen ( $\mathrm{Tr})$ Temperatur wurde anhand des gerätespezifischen Polynoms berechnet und bei der Peak-Temperaturangabe berücksichtigt.

$$
\mathrm{Tp}-\mathrm{Tr}=0,36+0,0037 \cdot \mathrm{T}+0,65 \cdot \mathrm{V}
$$

T: Grad Celsius, V: Aufheizgeschwindigkeit $\left(10^{\circ} / \mathrm{min}\right)$. 
drei bis vier deutlich getrennte Peaks $(587 \mathrm{~K}, 615 \mathrm{~K}, 682 \mathrm{~K}, 782 \mathrm{~K})$ und eine Schulter bei $566 \mathrm{~K}$ zu beobachten. Alternde Kulturen derselben Alge zeigen leicht veränderte Zusammensetzung.

Zwischen Trockengewicht und Energieinhalt besteht ein gesicherter linearer Zusammenhang. Die Proben aus der lag-Phase der Algenkultur zeigen pro Trockensubstanzeinheit leicht erhöhte Verbrennungsenergien. Im Tag-Nacht-Rhythmus ändert das Verhältnis Energie zu Trockensubstanz geringfügig, da die Trockensubstanz während der Nacht deutlich abnimmt, die Energie jedoch etwa auf gleichem Niveau bleibt. Dieses Verhalten lässt auf die Synthese energiereicher Verbindungen unter Ausnutzung der in der Kulturlösung gelösten organischen Stoffe schliessen. Der Verlauf der gelösten organischen Kohlenstoffverbindungen (Exkrete der Algen) entspricht dieser Hypothese: Während der Assimilationstätigkeit wird ein Anstieg, während der Dunkelphase meist eine deutliche Verringerung der Konzentration in der Lösung festgestellt.

\section{Schlussfolgerungen}

Die Ergebnisse der vorliegenden Energiemessungen zeigen, dass die Anteile der verschiedenen Stoffgruppen in den Zellen der gleichen Art als Folge unterschiedlicher Kulturbedingungen variieren. Zwischen grösseren taxonomischen Einheiten und zwischen Pflanzen und Tieren sind noch grössere Unterschiede zu erwarten. Unter der Voraussetzung einer vollständigen Verbrennung der gesamten organischen Substanz sind mikrokalorimetrische Daten eine Alternative zur heute praktizierten Biomassebestimmung über Volumenfaktoren. Sie gestatten die Berechnung der Energieverwertung bei Primär- und Sekundärproduzenten wie bei Destruenten und können zur Kalibrierung der Produktionsmessungen herangezogen werden.

Die Rolle der ins Wasser abgegebenen Stoffwechsel-Zwischenprodukte und Autolyseprodukte (summarisch als gelöster organischer Kohlenstoff DOC gemessen) ist noch unklar. Einerseits können diese Stoffe als Energiespender bzw. als Baustoff dienen, anderseits wirken sie als Vitamin bzw. Hemmstoff auf das Wachstum ein. Die vorliegenden Versuche lassen den Schluss zu, dass die Funktion als Energieträger sehr wesentlich sein kann. Eine eingehende Analyse des DOC und seiner Beziehung zu den einzelnen Trophiestufen drängt sich daher auf.

\section{SUMMARY}

Contribution to the problem of energy measurement in aquatic ecosystems: Microcalorimetric siudies of planktonic algae

A microcalorimetric method was applied in measuring the combustion enthalpy of the filter residue from a batch culture of Pediastrum boryanum. At the end of the dark resp. light phase, six parallel probes were filtered on a silver-membrane filter. For the energy measurement, two were programme-heated in an argon atmosphere and four in oxygen.

The precision of the method allows the measurement of $0.07 \mathrm{mg}$ dry weight with a tolerance of $\pm 8 \%$; whereby the precision of the apparatus is not yet at it's maximum. Through a consequent standardization of the various manipulations, even greater sensitivity can be attained.

The combustion of the substances begins at $450 \mathrm{~K}$ and ends at approx. $800 \mathrm{~K}$. Three or four separate peaks $(587 \mathrm{~K}, 615 \mathrm{~K}, 682 \mathrm{~K}, 782 \mathrm{~K})$ and a shoulder by $566 \mathrm{~K}$ are clearly distinguishable. Aging cultures of the same strain show a slightly varying composition. 
A clear linear relation exists between dry weight and energy content, whereby the regression line does not exactly intersect zero. The probes from the lag-phase of the algae cultures have a slightly higher energy content per dry weight unit. During the day-night rhythm the ratio energy to dry weight changes slightly, because the dry weight decrease during the night, while the energy content remains practically constant. This behaviour leads to the conclusion that energy-rich compounds are synthesized from solute organic compounds in the culture medium. The development of the dissolved organic compounds conform to this hypothesis: During the time of assimilation (light phase) an increase in the concentration of the DOC and during the dark phase a marked decrease (with one exception) is observed.

\section{RÉSUMÉ}

Contribution au problème de mesure d'énergie dans les écosystèmes aquatiques: Une recherche des algues planctoniques par un microcalorimètre

Une méthode microcalorimétrique a été appliquée pour mesurer l'enthalpie de combustion du résidu filtré d'une culture stationnaire de Pediastrum boryanum. A la fin de chaque phase obscure, resp. claire, six échantillons parallèles ont été filtrés sur une membrane d'argent. Pour la mesure d'énergie, deux d'entre eux ont subi un échauffement programmé dans une atmosphère d'argon et quatre autres dans l'oxygène.

La sensibilité de la méthode permet de mesurer l'énergie de $0,07 \mathrm{mg}$ de matière sèche avec une tolérance de $\pm 8 \%$, par quoi la précision de l'appareil n'est pas encore exploitée à fond. Par une standardisation adéquate des diverses manipulations, la sensibilité peut être améliorée.

La combustion des substances commence à $450 \mathrm{~K}$ et se termine à environ $800 \mathrm{~K}$. On peut observer trois à quatre «peaks» bien distincts $(587 \mathrm{~K}, 615 \mathrm{~K}, 682 \mathrm{~K}, 782 \mathrm{~K})$ et une «shoulder» à $566 \mathrm{~K}$. Les cultures vieillissantes des mêmes algues montrent un léger changement dans leur composition.

Il existe une nette relation linéaire entre le poids sec et le contenu énergétique. Les échantillons de la phase de latence de la culture d'algues ont une énergie de combustion légèrement supérieure par unité de poids sec. Pendant la période rythmée jour-nuit, le rapport entre l'énergie et le poids sec varie légèrement, car le poids sec diminue pendant la nuit, tandis que le contenu énergétique reste pratiquement constant. Ce comportement mène à la conclusion que les composés riches en énergie sont synthétisés à partir des matières organiques dissoutes dans le milieu de culture. Le développement des composés organiques du carbone dissous confirme cette hypothèse. On observe, pendant le temps d'assimilation, une augmentation, et pendant la phase obscure, une diminution marquée de la concentration de COD.

\section{VERDANKUNG}

Die Experimente wurden vom Schweizerischen Nationalfonds zur Förderung der wissenschaftlichen Forschung in verdankenswerter Weise unterstützt. Danken möchten wir auch Herrn Prof. Dr. H. Ambühl für die kritische Überarbeitung des Manuskripts und Frl. Heidi Bolliger für die Reinzeichnung der Abbildungen.

\section{LITERATURVERZEICHNIS}

1 Cummins, K.W., und Wuycheck, J.C.: Caloric Equivalents for Investigations in Ecological Energetics. Mitt. int. Ver. Limnol. 18, 1-158 (1971).

2 Gavrieli, J.: The Determination of $\mathrm{K}_{\mathrm{s}}$ for Ortho-Phosphate and Nitrate for Pediastrum boryanum using the Droop and Caperon Models for Steady State Growth of Algae. Interner Bericht EAWAG (1979).

3 Gnaiger, E.: Energetics of Invertebrate Anoxibiosis: Direct Calorimetry in Aquatic Oligochaetes. FEBS Lett. $112 / 2(1980)$.

4 Hemminger, W., und Hoehne, G.: Grundlagen der Kalorimetrie. Verlag Chemie, Weinheim 1979.

5 Jütner, F., und Matuschek, T.: The release of low molecular compounds by the Phytoplankton in an eutrophic lake. Water Res. 12, 251-255 (1978). 
6 Lamprecht, I., und Schaarschmidt, B.: Application of calorimetry in life sciences. Walter de Gruyter, Berlin 1977.

7 Lloyd, D.: Darkrespiration. In: Stewart, W.D.P.: Algal Physiology and Biochemistry. Ḃotanical Monographs, Bd. 10, S. 505-529. Blackwell, London 1974.

8 Müller, R., und Widemann. O.: Die Bestimmung des Nitrat-Ions in Wasser. Vom Wass. 22,247-271 (1955).

9 Redfield, A.C., Ketchum, B.H., und Richards, F.A.: The influence of organisms on the composition of sea-water. The Sea 2 (1963).

10 Sandermann, W., und Augustin, H.: Die thermische Zersetzung von Holz. Holz als Rohstoff 21, H.8 (1963).

11 Szabó, E., und Illi, R.: Die automatische Bestimmung von Phosphorverbindungen in Wasser. In Vorbereitung (1981).

12 Szabó, E., und Illi, R.: Die automatische Bestimmung des anorganischen und organisch gelösten Kohlenstoffs. In Vorbereitung (1981).

13 Tolbert, N.E.: Photorespiration. In: Stewart, W.D.P.: Algal Physiology and Biochemistry. Botanical Monographs, Bd. 10, S. 474-504. Blackwell, London 1974.

14 Utermoehl, H.: Zur Vervollkommnung der quantitativen Phytoplankton-Methodik. Mitt. int. Ver. Limnol. 2, 1-38 (1958).

15 Vogler, P.: Beiträge zur Phosphatanalytik in der Limnologie. II. Die Bestimmung des gelösten Orthophosphates. Fortschr. Wasserchem. Grenzgeb. 2, 109-119 (1965).

16 Vogler, P.: Die getrennte quantitative Bestimmung von gelösten Orthophosphatsäureestern ( «COPPhosphat») und gelösten kondensierten Phosphaten («POP-Phosphat»). Limnologica, Berlin 7, 309324 (1970).

Adresse der Autoren: EAWAG, Abteilung Hydrobiologie-Limnologie, Überlandstrasse 133, CH-8600 Dübendorf, Schweiz. 